\title{
Acoustic Emission Technique Applied in Textiles Mechanical Characterization
}

\author{
Carlos Rolando Rios-Soberanis ${ }^{1}$, Jose Rodriguez-Laviada ${ }^{1}$, Emilio Perez-Pacheco ${ }^{2}$, Takenobu Sakai ${ }^{3}$ and Shuichi \\ Wakayama $^{4}$
}

${ }^{1}$ Centro de Investigación Científica de Yucatán, Mérida, Yucatán, México

${ }^{2}$ Instituto Tecnológico Superior en el Estado de Campeche (ITESCAM), Calkini, Campeche, México

${ }^{3}$ Graduate School of Science and Engineering, Saitama University, Saitama, Japan

${ }^{4}$ Department of Mechanical Engineering, Tokyo Metropolitan University, Japan

\begin{abstract}
The common textile architecture/geometry are woven, braided, knitted, stitch boded, and Z-pinned. Fibres in textile form exhibit good out-of-plane properties and good fatigue and impact resistance, additionally, they have better dimensional stability and conformability. Besides the nature of the textile, the architecture has a great role in the mechanical behaviour and mechanisms of damage in textiles, therefore damage mechanisms and mechanical performance in structural applications textiles have been a major concern. Mechanical damage occurs to a large extent during the service lifetime consequently it is vital to understand the material mechanical behaviour by identifying its mechanisms of failure such as onset of damage, crack generation and propagation. In this work, textiles of different architecture were used to manufacture epoxy based composites in order to study failure events under tensile load by using acoustic emission technique which is a powerful characterization tool due to its link between $\mathrm{AE}$ data and fracture mechanics, which makes this relation a very useful from the engineering point of view.
\end{abstract}

\section{Introduction}

In order to predict the mechanical behavior of a composite during its service life, it is important to study the initiation and development of cracks and its effects induced by degradation. The onset of damage is related to the structural integrity of the component and its fatigue life. For this, among other reasons, non-destructive techniques have been widely used nowadays in composite materials characterization such as acoustic emission. Acoustic Emission (AE) analysis is an extremely powerful technology that can be deployed within a wide range of application of non-destructive testing: metal pressure vessels, piping systems, reactors and similar [1]-[4]. However lately, AE technique has been used extensively in materials characterization, especially on composite materials research by investigating properties of materials, breakdown mechanisms and damage behavior [5]-[7]. AE technique is the most powerful method since it correlates $\mathrm{AE}$ signals detected intimately to materials fracture mechanics, which makes this relation a very useful tool from the engineering point of view [7]. In this context, AE can also monitor the damage onset and the damage evolution as well as its profile in real time data, which can assess the structural integrity of the component under loading. Damage mechanisms up to failure are rather complex in composite laminates [8]. One reason is that several damages phenomena (matrix cracking, delamination, fibre breakage, fibre/matrix debonding, fibre pull-out, etc.) are acting alone or coupled. The identification and evaluation of damage or fracture stages of polymer composites are extremely complex due to a composite material exhibiting heterogeneous nature, lacking of uniformity in composition owing to its components and, therefore, the assignment may be controversial [9], [10]. The new advanced technologies in textile industry has allowed the emerge of novel fabric geometries used for composites reinforcement in addition to those traditionally produced. When textiles are used as reinforcements in composite materials, they exhibit very heterogeneous structures As a consequence; the stress and strain fields within it depend greatly on the constituents repartition, on their size and on their spatial arrangement [11]-[13]. In other words, it depends on the textile internal geometry. Hence, a characterization procedure must be able to deal with this geometrical heterogeneity and this mechanical anisotropy. This paper focuses in AE damage analysis in diverse textiles of different architecture/geometry, therefore, damage sequence and its dependence is discussed.

\section{Case 1. Damage analysis in multiaxial textile reinforced composites}

A multi-axial E-glass textile exhibiting a mass per unit area of $972 \pm 5 \% \mathrm{~g} / \mathrm{m} 2$ and a $\left[0^{\circ},+45^{\circ}, 90^{\circ},-45^{\circ}\right]$ stacking 
sequence was used as a reinforcement in epoxy resin matrix composite. The layers have relative mass fractions as indicated in Table 1 and are stitched together with a polyester multifil binding yarn. The unidirectional fiber bundles in the four planes are held together by a fine polyester thread in both sides. In the outer layer where fibers are running at $0^{\circ}$, the polyester (PES) thread is knitted in a zig-zag pattern and in the other side (outer layer at $-45^{\circ}$ ) is knitted at $0^{\circ}$ (Fig. 1).

Table 1. Technical specification of the textile.

\begin{tabular}{|c|c|c|}
\hline Layer orientation & Weight $\mathbf{g} / \mathbf{m}^{2}$ & Composition \\
\hline $0^{\circ}$ & 354 & E- Glass \\
\hline$+45^{\circ}$ & 200 & E- Glass \\
\hline $90^{\circ}$ & 207 & E- Glass \\
\hline$-45^{\circ}$ & 200 & E- Glass \\
\hline Knity arn & 11 & Polyester \\
\hline Total & $972 \pm 5 \%$ & \\
\hline
\end{tabular}
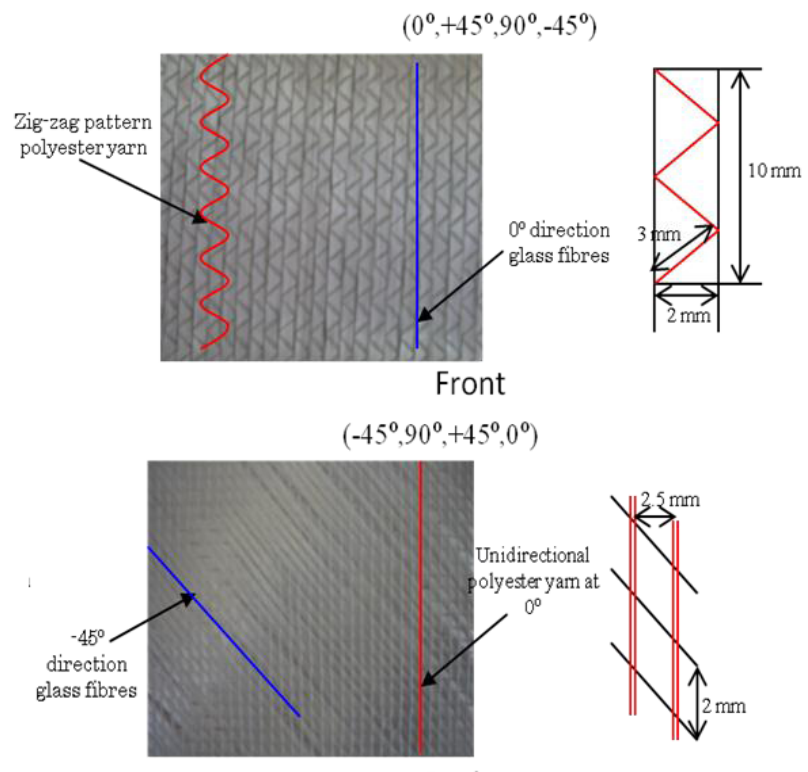

Back

Figure 1. Textiles interweaving, front and back.

Fig. 2 illustrates both sides of the Z-pinned multiaxial textile. Epoxy system chosen was completely transparent when cured allowing visualization of the textiles architecture. Using one layer of textile and epoxy resin system as matrix, a composite with a fibre volume fraction of about $18 \%$ was manufactured by wet lay-up process. Laminas were elaborated taking in account the two principal direction layers that conform the multiaxial textile $\left(0^{\circ}\right.$, and $\left.90^{\circ}\right)$.
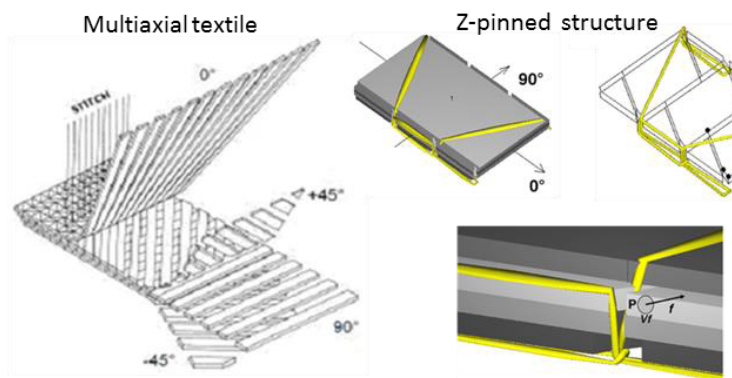

Figure 2. Z-pinned multiaxial textile architecture/geometry.
A Shimadzu universal machine was used to carry out mechanical tests in tension. The textile direction in question was parallel to load direction in which the samples were loaded to progressively higher strain at a cross-head speed of $0.5 \mathrm{~mm} / \mathrm{min}$. Acoustic emission sensors were attached to the sample as shown in Fig. 3A to obtain the signal in order to be related to de damage development and mechanisms of fracture.

A
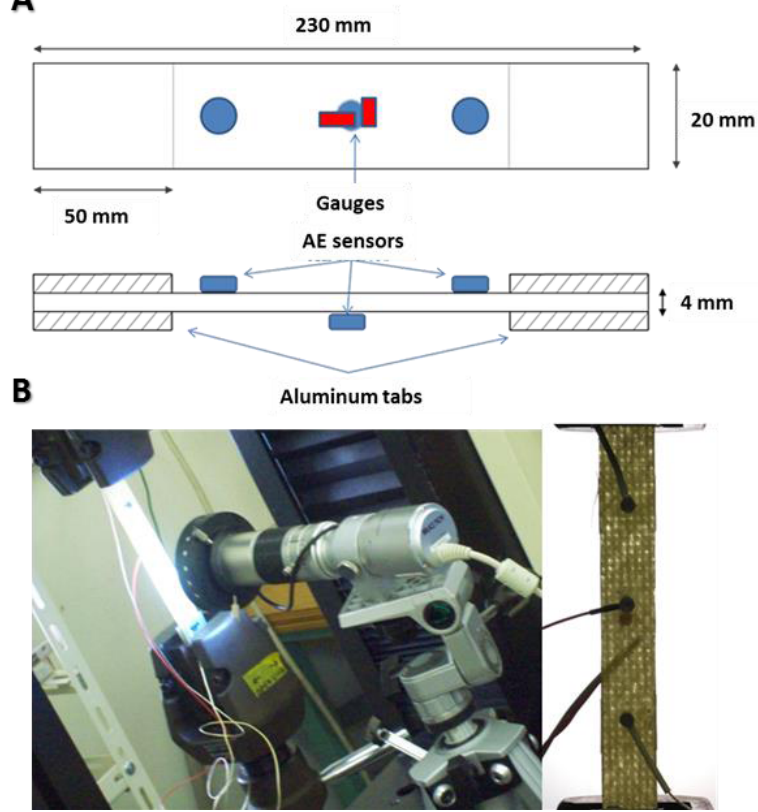

Figure 3. A) Sample dimensions, B) load recording in tension.

During tensile loading, in each stress-strain curves, several discontinuities that are related to significant cracks developed transversally to the sample were observed. At a higher strain discontinuities were more frequent, the higher the load, the more cracks are formed. Samples at $90^{\circ}$ exhibited slightly higher crack density (cracks per area). Apparently, textile geometry (stacking sequence) does not affect significantly the mechanical parameters values; however, damage development and propagation along samples depending on the loading direction are influenced by the PES knit yarn structure (Fig. 4).
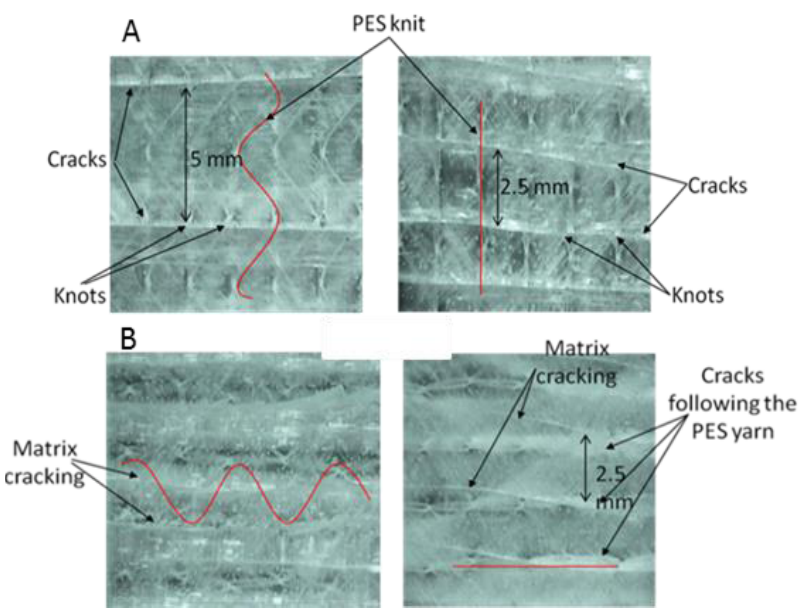

Figure 4. A) Textile oriented at $0^{\circ}$, B) Textile oriented at $90^{\circ}$.

The load (N)-time (s)-amplitude curves for samples when the textile is oriented at $0^{\circ}$ is presented in Fig. $5 \mathrm{~A}$. 
AE signal detected over $280 \mathrm{~s}$ are intimately related to the first deflection in the curve which is also correlated to the appearance of the first significant damage that emits high energy.
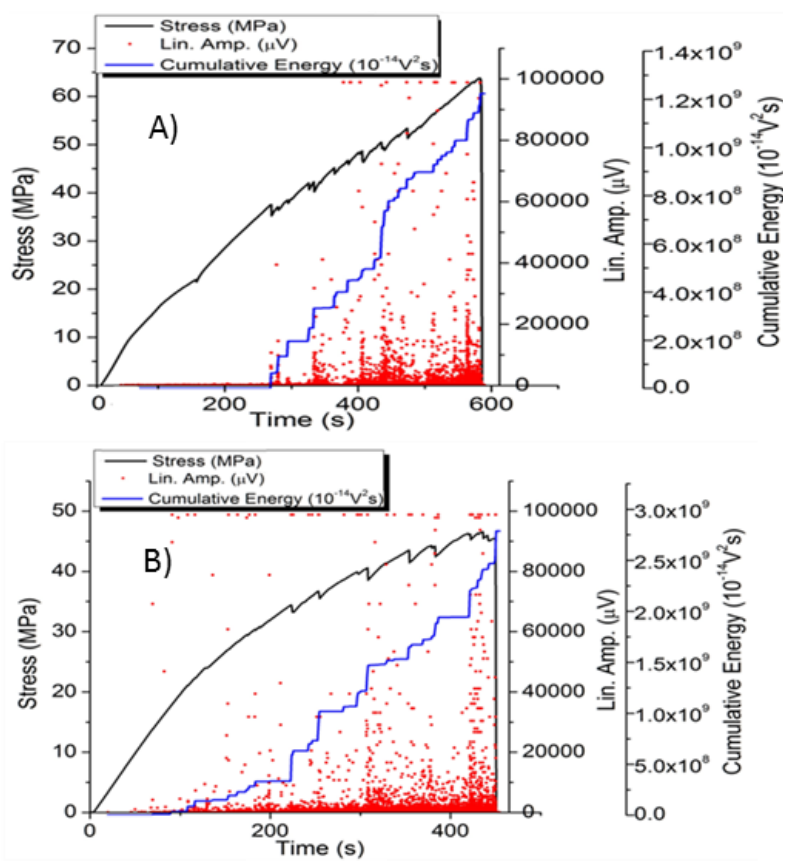

Figure 5. A) Textile oriented at $0^{\circ}$, B) Textile oriented at $90^{\circ}$.

This damage is associated to matrix cracking in the composite. At $90^{\circ}$ direction (Fig. 5B), is possible to observe more small discontinuities related with the $\mathrm{AE}$ signals identified as matrix cracking. Energy is augmented rapidly as the more cracking damage occurs. In all the samples tested in tension $\left(0^{\circ}\right.$, and $\left.90^{\circ}\right)$, no fiber breakage was observed or AE detected until total fracture happened. Samples at $90^{\circ}$ exhibited an early damage development in comparison with $0^{\circ}$. Lower damage initiation at $90^{\circ}$ samples apparently was due to the major amount of unidirectional glass fibres at $0^{\circ}$ layer (denser) that is positioned perpendicular to the applied stress and allows more transversal matrix cracks to be developed along the coupon.

\section{Case 2.- Mechanical properties of geotextiles: effect of architecture}

Two geotextiles of different geometry were used to manufacture a polymeric matrix based composite material. First one is made of polyethylene-terephthalate (PET) fibers constituted by unidirectional threads (woven) oriented in longitudinal and transversal directions. Transversal yarns are wider presenting higher amount of filaments than in longitudinal direction. Second one is a polypropylene (PP) based geotextile constituted by a diagonal longitudinal "tape like" and transversal yarns having an oval shape in the intersections (Fig. 6). It is observed that this textile exhibits imperfections in the surface such a microcracks. In order to observe the onset and development of damage to identify the fracture progression, the textiles were placed in epoxy matrix to manufacture a composite material.
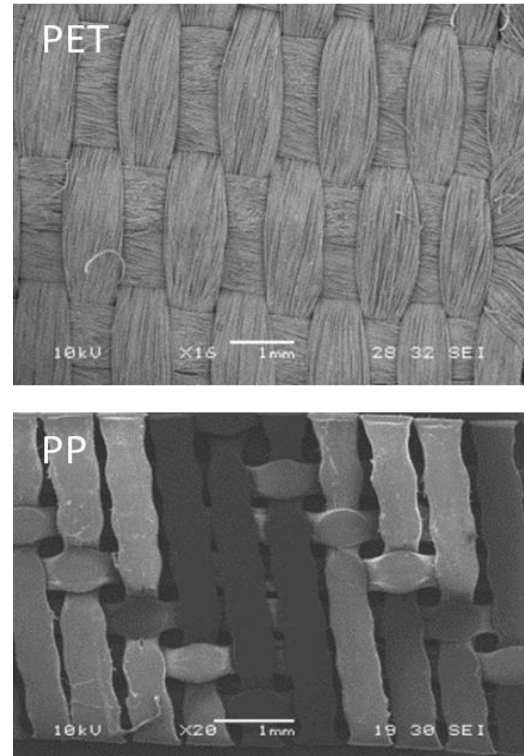

Figure 6. Geotextiles architecture (PET and PP).

Geotextiles were mechanically characterized individually in tension mode in both longitudinal $\left(0^{\circ}\right)$, and transversal direction $\left(90^{\circ}\right)$, in order to compare their mechanical behavior. Composite materials were tensile tested in a Universal Machine Shimadzu using $5 \mathrm{kN}$ load cell at $0.1 \mathrm{~mm} / \mathrm{min}$ in order to observe the crack progression along the sample. Acoustic Emission sensors were attached to the composite samples in order to monitor damage in "real time "as seen in Fig. 3.

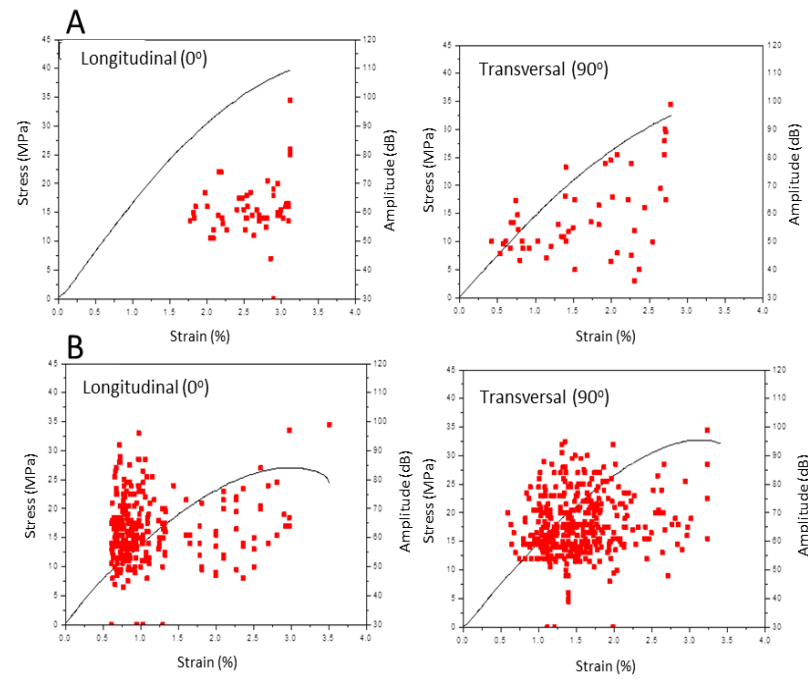

Figure 7. A) Epoxy/PET geotextile composites, B) Epoxy/PP geotextile composites.

Results of mechanical behavior in tensile test coupled to acoustic emission analysis for epoxy/geotextile composites are shown in Fig. 7. Fig. 7A shows the stressstrain behavior of epoxy/PET geotextile composite tested in longitudinal and transversal direction respectively. In longitudinal direction, first EA signals are sensed at about $1.75 \%$ strain with high amount of signals with high amplitude (50-80 dB) less disperse. Such signals can be correlated to interfacial damage and matrix crack progression. On the other hand, stress-strain curve when epoxy/PET geotextile is tested in transversal direction, 
AE signals were detected significantly sooner compared with the longitudinal sample. Stress-strain curve for epoxy/PP geotextile composite tested in longitudinal and transverse directions are presented in Fig. 7B. In composites tested longitudinally, AE signals showed up at lower strains $(0.6 \%)$ after linearity but with higher amplitude. Higher cracking density was found between 0.7 and 1.2 that may be related to the extensive crack progression generated by the complex textile geometry. Mechanical tendency observed for epoxy/PP geotextile composites tested in transversal direction is somehow similar to longitudinal samples, however, transversal samples showed higher density of AE signals of significant amplitude. This effect may be related to the fact that this direction presented major stress resistance, therefore it requires more load and consequently, the damage generated in the composite can be larger.

\section{Conclusions}

In both cases, it has been demonstrated that architecture/geometry of the textiles are an essential factor that influences the mechanical behavior of a composite material and that it is dependent on the loading orientation. Damage cracking initiation, development and progression are governed by the way in which fiber bundles are interweaved. In Case 1, damage cracking development was affected by the polyester thread (zigzag and unidirectional). It was demonstrated the influence of the textile stitched geometry in the initiation sites and the damage development of the composite. Zig-zag polyester knit yarn was observed to govern the transversal crack development while unidirectional $\left(0^{\circ}\right)$ polyester thread only influenced damage by the stitch knots and when it was oriented transversally to load direction. In the $90^{\circ}$ direction, transversal flaws were formed due to fibre/matrix debondings that coalesce at higher strain to generate the significant cracks. These visible cracks have a nearly equidistant spacing and are equally distributed in the $+45^{\circ}$ and $-45^{\circ}$ plies. First AE signals detected during tensile tests were identified as damage induced in the material at the fibre/matrix interphase and demonstrated how damage initiation is affected by NCF orientation. In Case 2, mechanical properties were studied in geotextiles made of polyethylene terephthalate (PET) and polypropylene (PP), both textiles with different architecture/geometry of waving. Acoustic emission signals in epoxy/PP geotextile composite exhibited higher density in comparison to epoxy/PET geotextile composite. This effect indicates that epoxy/PP geotextile composite generates more damage events during tensile tests which are intimately related to the complexity of threads architecture/geometry that originates active concentrations sites that release energy when having internal damage. Finally, it was shown that Acoustic Emission technique is a powerful characterization tool for understanding materials mechanical response under any type of external load due to its link between AE data and fracture mechanics, which makes this relation a very useful for identification of damage sequence.

\section{Acknowledgement}

The author is grateful to the Mexican Council for Science and Technology (CONACYT) for the provision of grant 60204/ CM0042.

\section{References}

1. G. Du, J. Li, W.K. Wang, C. Jiang, S.Z. Song, Corrosion Science, 53, Issue 9, 2918-2926, (2011).

2. P. Nivesrangsan, J. A. Steel, R.L. Reuben, Mechanical Systems and Signal Processing, 21, Issue 2, 1103-1114, (2007)

3. G.C. McLaskey, S.D. Glaser, C.U. Grosse, Journal of Sound and Vibration, 329, no. 12, 2384-2394, (2010)

4. D.G. Aggelis, S. Verbruggen, E. Tsangouri, T. Tysmans, D. Van Hemelrijck, Construction and Building Materials, 47, 1037-1045, (2013)

5. C.R. Ramirez-Jimenez, N Papadakis, N Reynolds, T.H Gan, P Purnell, M Pharaoh, Composites Science and Technology, 64, no. 12, 1819-1827 (2004)

6. M. Wevers, NDT \& E International, 30, no. 2, 99106, (1997)

7. R. Gutkin, C.J. Green, S. Vangrattanachai, S.T. Pinho, P. Robinson, P.T. Curtis, Mechanical Systems and Signal Processing, 25, Issue 4, 1393-1407, (2011)

8. O.Y. Andreykiv, M.V. Lysak, O.M. Serhiyenko, V. R. Skalsky, Engineering Fracture Mechanics, 68, Issue 11, 1317-1333, (2001)

9. I.M. De Rosa, C. Santulli, F. Sarasini, Composites Part A: Applied Science and Manufacturing, 40, no. 9, 1456-1469, (2009)

10. H. Ku, H. Wang, N. Pattarachaiyakoop, M. Trada, Composites Part B: Engineering, 42, no. 4, 856-873, (2011)

11. Y.H. Yu, J.H. Choi, J.H. Kweon, D.H. Kim, Composite Structures, 75, Issues 1-4, 163-169, (2006)

12. N. Kosmann, J.M. Karsten, M. Schuett, K. Schulte, B. Fiedler, Composites Part B: Engineering, 70, 184188, (2015)

13. L. Lia, S.V. Lomov, X. Yan, Composite Structures, 123, 45-53, (2015) 